\title{
Size effect of PLGA spheres on drug loading efficiency and release profiles
}

\author{
G. J. S. Dawes · L. E. Fratila-Apachitei - K. Mulia • \\ I. Apachitei · G.-J. Witkamp · J. Duszczyk
}

Received: 1 September 2008/Accepted: 9 December 2008/Published online: 22 January 2009

(C) The Author(s) 2009. This article is published with open access at Springerlink.com

\begin{abstract}
Drug delivery systems (DDS) based on poly (lactide-co-glycolide) (PLGA) microspheres and nanospheres have been separately studied in previous works as a means of delivering bioactive compounds over an extended period of time. In the present study, two DDS having different sizes of the PLGA spheres were compared in morphology, drug (dexamethasone) loading efficiency and drug release kinetics in order to investigate their feasibility with regard to production of medical combination devices for orthopedic applications. The loaded PLGA spheres have been produced by the oil-in-water emulsion/solvent evaporation method following two different schemes. Their morphology was assessed by scanning electron microscopy and the drug release was monitored in phosphate buffer saline solution at $37^{\circ} \mathrm{C}$ for $550 \mathrm{~h}$ using high performance liquid chromatography. The synthesis schemes used produced spheres with two different and reproducible size ranges $(20 \pm 10$ and $1.0 \pm 0.4 \mu \mathrm{m})$ having a smooth outer surface and regular shape. The drug loading efficiency of the $1.0 \mu \mathrm{m}$ spheres was found to be $11 \%$ as compared to just $1 \%$ for the $20 \mu \mathrm{m}$ spheres. Over the $550 \mathrm{~h}$ release period, the larger spheres (diameter $20 \pm 10 \mu \mathrm{m}$ ) released $90 \%$ of the encapsulated dexamethasone in an approximately linear fashion whilst the relatively small spheres
\end{abstract}

G. J. S. Dawes · L. E. Fratila-Apachitei $(\bowtie)$ · I. Apachitei ·

J. Duszczyk

Department of Materials Science and Engineering,

Delft University of Technology, Mekelweg 2,

2828 CD Delft, The Netherlands

e-mail: E.L.Fratila-Apachitei@tudelft.nl

K. Mulia · G.-J.Witkamp

Laboratory for Process Equipment, Delft University

of Technology, Leeghwaterstraat 44, 2628 CA Delft,

The Netherlands (diameter $1.0 \pm 0.4 \mu \mathrm{m}$ ) released only $30 \%$ of the initially loaded dexamethasone, from which $20 \%$ within the first $25 \mathrm{~h}$. The changes observed were mainly attributed to the difference in surface area between the two types of spheres as the surface texture of both systems was visibly similar. As the surface area per unit volume increases in the synthesis mixture, as is the case for the $1.0 \mu \mathrm{m}$ spheres formulation, the amount of polymer-water interfaces increases allowing more dexamethasone to be encapsulated by the emerging polymer spheres. Similarly, during the release phase, as the surface area per unit volume increases, the rate of inclusion of water into the polymer increases, permitting faster diffusion of dexamethasone.

\section{Introduction}

Poly (lactide-co-glycolide) (PLGA) has been studied for many years as a suitable drug delivery material, mainly due to its chemical biocompatibility [1,2], total biodegradability, and non-toxic degradation products [3]. Because of these factors it can be also considered for the production of medical combination devices with microtextured metallic implants (drug/device), such as drug eluting orthopedic implants. A micro- or nano-spherical matrix for drug delivery is preferred in this case as the texture of the metallic biomaterial can be maintained and potentially the drug release profile can be extended over longer durations relative to other formulations such as films. However, in order to consider such a system, the individual components must first be assessed.

A common method for the sustained release of drugs is the production of a PLGA microsphere formulation by the oil-in-water emulsion and solvent evaporation technique, 
where the drug is evenly distributed throughout a soluble PLGA matrix and then emulsified. Once the organic solvent evaporates off, a suspension of solid microparticles is left. The bioactive can be co-dissolved in the PLGA if hydrophobic [4], or produced as a double emulsion or suspension if not [5]. The hydrophilicity of this polymer is defined by the lactide:glycolide ratio and can be used to alter the release rate in a microparticulate formulation [6]. The polymer molecular weight also influences the drug release rate [7]. The rate of degradation is inversely proportional to the proportion of lactide monomer in the polymer strands [1], and the rate of diffusion of drugs through the polymer matrix inversely proportional to the molecular weight of the PLGA polymer.

Microscale spheres are typically used in applications where the drugs must be delivered to only one site without diffusing away, such as the reduction of inflammation near glucose sensors [8], controlled release of insulin [9], or delivery of morphogenetic proteins for bone growth [10]. It has been considered [1] that the most effective size range for this is $10-200 \mu \mathrm{m}$. Where smaller than $10 \mu \mathrm{m}$, the microspheres become subject to not only diffusion, but are able to be phagocytosed by immune cells. This has the two downsides of not only the immune cells being capable of transporting the microspheres away from their intended site, but due to the phagocytosis the microspheres are much more rapidly broken down inside the acidic vesicles causing rapid release and potentially negating the slow release effect. Using microspheres larger than $200 \mu \mathrm{m}$ may have a deleterious effect upon the tissue structure of the implantation site, potentially causing an increased immune response and unnecessary inflammation. Additionally, due to the increased radius and decreased surface area, the rate of water permeation and matrix degradation would be diminished, reducing the maximum possible rate of drug release.

The work undertaken in this paper was performed in order to assess the drug release kinetics and system efficiencies of two different PLGA sphere formulations (i.e. 20 vs. $1 \mu \mathrm{m}$ spheres) in the view of further research towards the application of these systems for orthopedic medical combination devices. In this case, the drug delivery systems would become an integral part of the device providing a sustained drug release from, for example, a titanium hip stem. The model drug used in this investigation, dexamethasone $\left(\mathrm{C}_{22} \mathrm{H}_{29} \mathrm{FO}_{5}\right)$, is a hydrophobic anti-inflammatory corticosteroid that is used to treat many inflammatory responses [8] and known to induce osteoblast differentiation [11]. The PLGA spheres loaded with dexamethasone have been produced by a similar method (oil-in-water emulsion/solvent evaporation) following two different schemes. Their morphology (size and shape), the drug loading efficiency and the drug release profiles in phosphate buffer saline solution at $37^{\circ} \mathrm{C}$ over a period of $550 \mathrm{~h}$ have been investigated.

\section{Materials and methods}

\subsection{Materials}

Poly (lactide-co-glycolide) (MW: 40,000-75,000, 50:50), dexamethasone (98\%), poly (vinyl alcohol) (MW: 30,00070,000), sodium azide, disodium hydrogen phosphate, sodium dihydrogen phosphate, acetic acid, sodium acetate and all solvents were purchased from Sigma Aldrich chemicals. Distilled, deionised water $\left(\mathrm{dd}_{\mathrm{H}} \mathrm{O}\right)$ was provided from Millipore.

Phosphate buffered saline (PBS) was made up as the simulated body fluid with the following concentrations: $0.24 \mathrm{~g} / \mathrm{l}$ potassium dihydrogenphosphate, $1.44 \mathrm{~g} / \mathrm{l}$ sodium hydrogenphosphate, $0.2 \mathrm{~g} / \mathrm{l}$ potassium chloride, $8 \mathrm{~g} / \mathrm{l}$ sodium chloride and $0.1 \mathrm{~g} / \mathrm{l}$ sodium azide at $\mathrm{pH}$ 7.0.

\subsection{Sphere synthesis}

\subsubsection{Scheme 1}

Spheres were synthesised following a modified version of the oil-in-water emulsion/solvent evaporation method used by T. Hickey [4]. $400 \mathrm{mg}$ PLGA and $80 \mathrm{mg}$ dexamethasone were co-dissolved in $40 \mathrm{ml}$ dichloromethane:methanol 9:1. $5 \mathrm{ml}$ fractions of this solution was added each to $100 \mathrm{ml}$ $0.2 \% \mathrm{w} / \mathrm{v}$ PVA solution in ${ }_{\mathrm{dd}} \mathrm{H}_{2} \mathrm{O}$, and stirred at $1,250 \mathrm{rpm}$ for $30 \mathrm{~min}$. The resulting emulsion was left to stir at $60 \mathrm{rpm}$ for a further $18 \mathrm{~h}$ so that the organic solvents could evaporate and let the spheres harden. The spheres were then recovered from solution by filtration, and washed on the filter with ${ }_{\mathrm{dd}} \mathrm{H}_{2} \mathrm{O}$ to remove excess PVA and unencapsulated dexamethasone. The resulting slurry was dried fully in a lyophiliser over $24 \mathrm{~h}$, producing a fine white powder.

\subsubsection{Scheme 2}

This scheme was aimed at synthesis of significantly smaller spheres. They were produced following a modified version of the route explored by Sahoo et al. [12]. About $500 \mathrm{mg}$ PLGA and $100 \mathrm{mg}$ dexamethasone were codissolved in $10 \mathrm{ml}$ dichloromethane:methanol 9:1. This solution was added to $60 \mathrm{ml}$ of $0.2 \% \mathrm{w} / \mathrm{v}$ PVA solution, and vortexed at 13,500 rpm for $6 \mathrm{~min}$. The resulting milky white emulsion was sonicated at $20 \mathrm{~W}$ for a further $5 \mathrm{~min}$, before being topped up to approx. $100 \mathrm{ml}$ with more $0.2 \%$ PVA, and allowed to stir at $60 \mathrm{rpm}$ overnight to harden. The resulting spheres were recovered by ultracentrifugation for $15 \mathrm{~min}$ at $18,000 \times g$. The supernatant was poured off, and the resulting solids washed with ${ }_{\mathrm{dd}} \mathrm{H}_{2} \mathrm{O}$, resuspended, and centrifuged a second time, in order to remove excess PVA and unencapsulated dexamethasone. The resulting slurry was freeze dried as above. 
2.3 Scanning electron microscopy (SEM) and size analysis

Pictures of each type of PLGA spheres were taken using a JEOL 5400 SEM at $10 \mathrm{kV}$ after gold sputter coating. These were then submitted to visual analysis by AnalySIS software in order to produce a size distribution for each sample. At least 150 data points were used to get an accurate distribution of sizes. After the drug release experiment, samples of each type of degraded PLGA sphere were analysed by SEM.

\subsection{Drug loading efficiency}

About $10 \mathrm{mg}$ of each dried sphere type was dissolved in approx. $0.7 \mathrm{~g}$ acetonitrile. These samples were run on a High Performance Liquid Chromatography (HPLC) machine HPLC fitted with a reverse phase Varian Chromsphere $\mathrm{C} 18$ column $(250 \times 4.6 \mathrm{~mm})$ with a running buffer of $60 \%$ acetonitrile, $40 \%$ sodium acetate buffer at $\mathrm{pH} 4.8$. The concentration of dexamethasone was resolved using a Varian Prostar spectrometer reading at $254 \mathrm{~nm}$, with a dexamethasone peak at $3.62 \mathrm{~min}$. The drug loading efficiency (DLE) was calculated using the following formula:

DLE $=\frac{\text { encapsulated drug }}{\text { maximum encapsulation }} \times 100 \%$

The maximum encapsulation was taken to be the point where all the supplied dexamethasone was encapsulated in the spheres.

\subsection{Release profile assay}

About $60 \mathrm{mg}$ of each sphere type were split between 3 separate samples of $25 \mathrm{ml}$ PBS at $\mathrm{pH} 7.0$ and incubated at $37^{\circ} \mathrm{C}, 30 \mathrm{rpm}$ for the length of sampling. Samples of $1.7 \mathrm{ml}$ were removed and filtered through a $220 \mathrm{~nm}$ polyester membrane to remove the PLGA spheres. The incubated sample was then topped up to $25 \mathrm{ml}$ with fresh, filtered PBS.
Each removed sample was run on an HPLC as described above, with a dexamethasone peak at $3.62 \mathrm{~min}$, and a peak corresponding to the simulated body fluid at $1.76 \mathrm{~min}$.

\section{Results and discussion}

\subsection{Spheres morphology}

SEM images of the spheres produced by the two different synthesis schemes are shown in Fig. 1. A significant difference in the size of the two types of spheres was obtained. The images were further processed for size distribution and the findings are included in Fig. 2. In addition, the particles revealed a smooth outer surface and a regular spherical shape. A low surface roughness is important for extended drug release. Any irregularities will cause an increase in surface area, creating a much faster diffusive release of dexamethasone.

It is visible from the SEM images that the small spheres (Fig. 1b) are more agglomerated and clumped showing a more powder-like arrangement. Their visualization by SEM was relatively more difficult due to a high degree of charging and sample melting under the electron beam.

From the size analysis results (Fig. 2), it can be observed that the two different synthesis schemes generated separate size ranges: $20 \pm 10 \mu \mathrm{m}$ and $1.0 \pm 0.4 \mu \mathrm{m}$, respectively. The distribution of sizes for the large sphere formulation was relatively broad and may be connected to the differences between the synthesis methods, for example the stirring speed. If the spheres were to be placed onto a metallic microtextured biomaterial, more of the underlying texture would be maintained when using the $1.0 \mu \mathrm{m}$ spheres.

\subsection{Drug loading efficiency}

The drug loading efficiency for the $1.0 \mu \mathrm{m}$ spheres was calculated to be $11.2 \%$ whereas for the $20 \mu \mathrm{m}$ spheres, $0.9 \%$. This difference is likely due to the increased emulsion
Fig. 1 Morphology of the PLGA spheres visualized by scanning electron microscopy: a synthesis scheme $1(10 \mathrm{kV}$, $\times 200)$; b synthesis scheme 2 $(10 \mathrm{kV}, \times 7,500)$
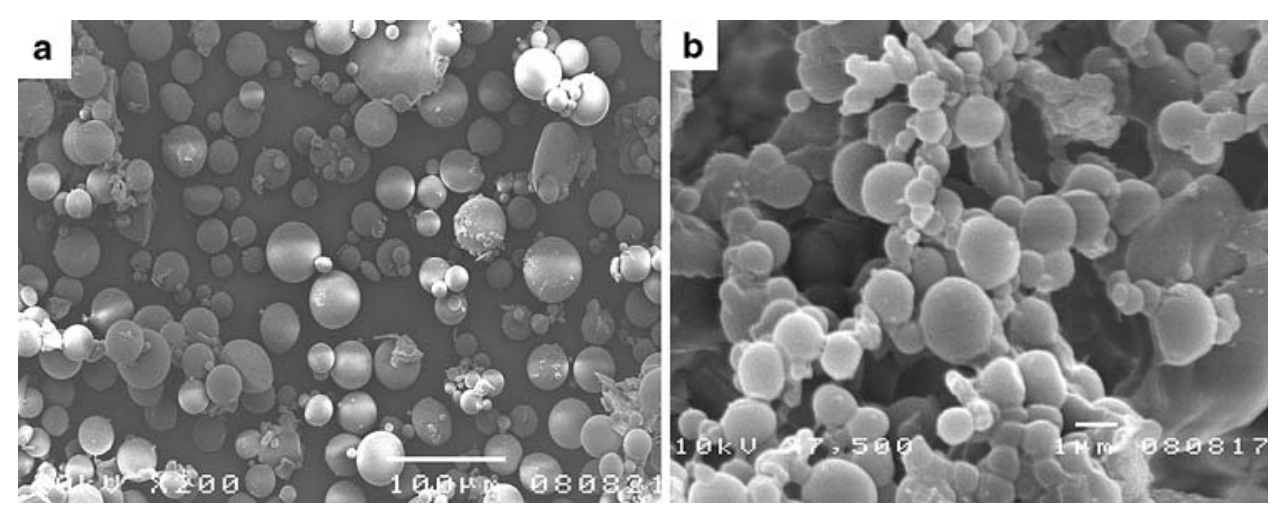
Fig. 2 PLGA spheres size for the two different synthesis schemes used. The values were calculated by visual analysis of the SEM images and include more than 150 data points for each series

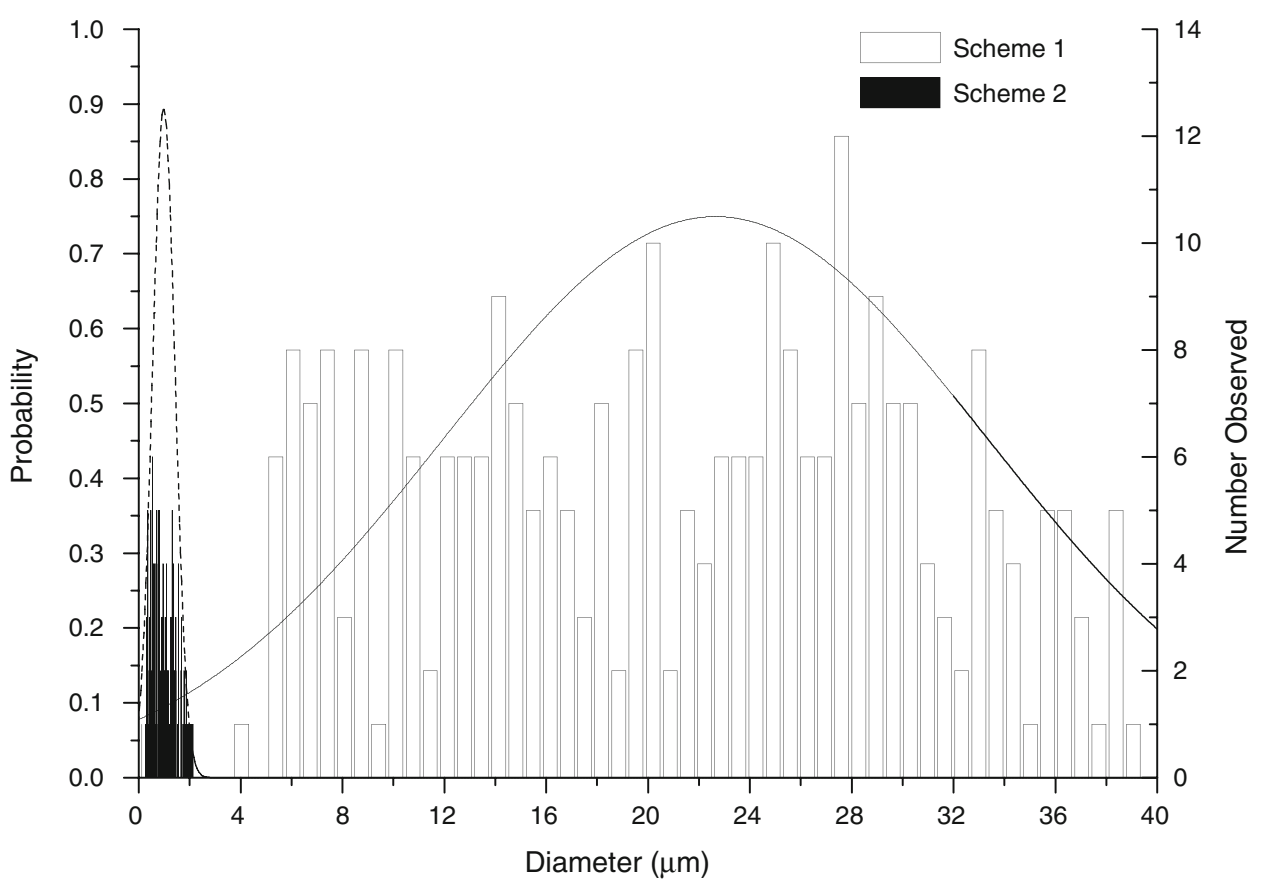

surface area generated in the second synthesis scheme used for the small spheres, allowing more of the dexamethasone to remain incorporated within the PLGA matrix as it forms. During the first scheme, much of the dexamethasone is released into the aqueous component, where it likely forms microcrystalline deposits due to its extremely low partition coefficient. These deposits are lost in the washing steps.

\subsection{Drug release profile}

Over the course of $550 \mathrm{~h}$ (Fig. 3), the $20 \mu \mathrm{m}$ spheres released $90 \%$ of their calculated yield of dexamethasone

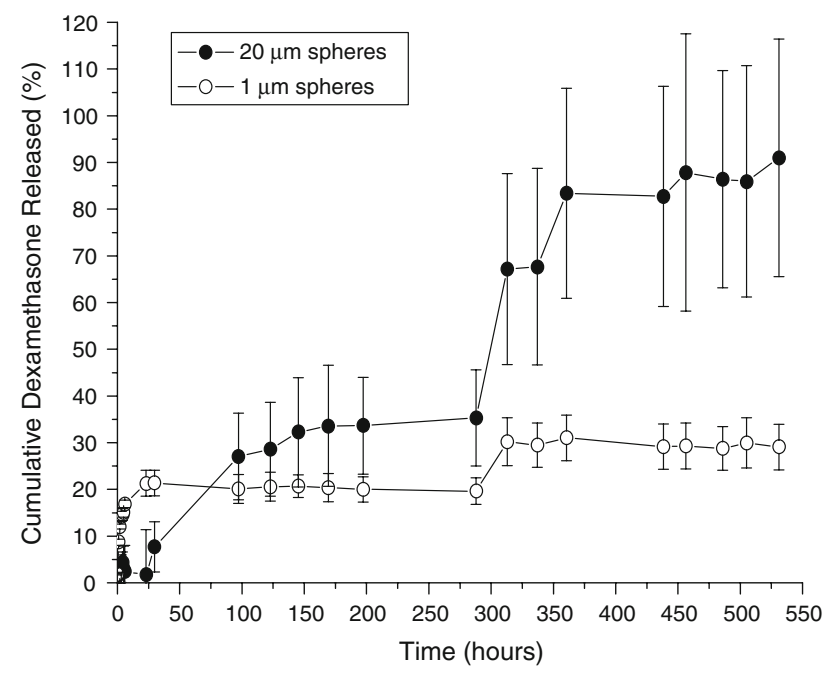

Fig. 3 Dexamethasone release profiles from the 20 and $1.0 \mu \mathrm{m}$ PLGA spheres (PBS, $\left.37^{\circ} \mathrm{C}\right)$. Average values with standard deviations are presented $(n=3)$ exhibiting a roughly linear profile, whilst the $1.0 \mu \mathrm{m}$ spheres released $30 \%$ out of which $20 \%$ in very early stages (less than $25 \mathrm{~h}$ ). A closer look at the release profile of the $20 \mu \mathrm{m}$ spheres suggests the existence of three distinctive zones, namely the first $150 \mathrm{~h}, 150-300 \mathrm{~h}$, and 300$550 \mathrm{~h}$. For the first $150 \mathrm{~h}$ the relatively high release rates may be attributed to diffusion of dexamethasone from the sphere subsurface. In the next $150 \mathrm{~h}$, the release slows drastically down to only $0.02 \% / \mathrm{h}$ suggesting a depletion of dexamethasone from within the available diffusion distance from the surface. In the last $250 \mathrm{~h}$ a climb in release to an average of $0.23 \% / \mathrm{h}$ was observed as the polymer is degraded following water inclusion into its structure thus allowing for a combined diffusion-degradation mechanism of dexamethasone release. Figures $4 \mathrm{a}$, b show SEM images of the spheres after degradation for $550 \mathrm{~h}$. A clear surface porosity for the spheres produced by scheme 1 , and also some degree of particle swelling was visible (Fig. 4a). The release profile follows the trend previously shown by Hickey et al. [4] for $11 \mu \mathrm{m}$ size PLGA spheres.

By going down with the size of the loaded spheres, significant changes occurred in the release profile of dexamethasone (Fig. 3). Initially, the $1.0 \mu \mathrm{m}$ spheres released dexamethasone at a rate of $5 \%$ per hour, but this fell to just $0.02 \% / \mathrm{h}$ after $25 \mathrm{~h}$, and this rate of release was held on average from $25 \mathrm{~h}$ until the end of the experiment. This is in line with the trends in release shown in the literature [13]. Such a rapid initial burst can be mostly associated with diffusion from the outermost layers of the particle surface, as in the case with the burst for the $20 \mu \mathrm{m}$ spheres. For the first $6 \mathrm{~h}$ the release for the $1.0 \mu \mathrm{m}$ spheres was much faster than the $20 \mu \mathrm{m}$ spheres, with a rate of 
Fig. 4 Morphology of the PLGA spheres after $550 \mathrm{~h}$ degradation, visualized by scanning electron microscopy: a $20 \mu \mathrm{m}$ spheres $(10 \mathrm{kV}$, $\times 2,000)$; b $1 \mu \mathrm{m}$ spheres $(10 \mathrm{kV}, \times 7,500)$
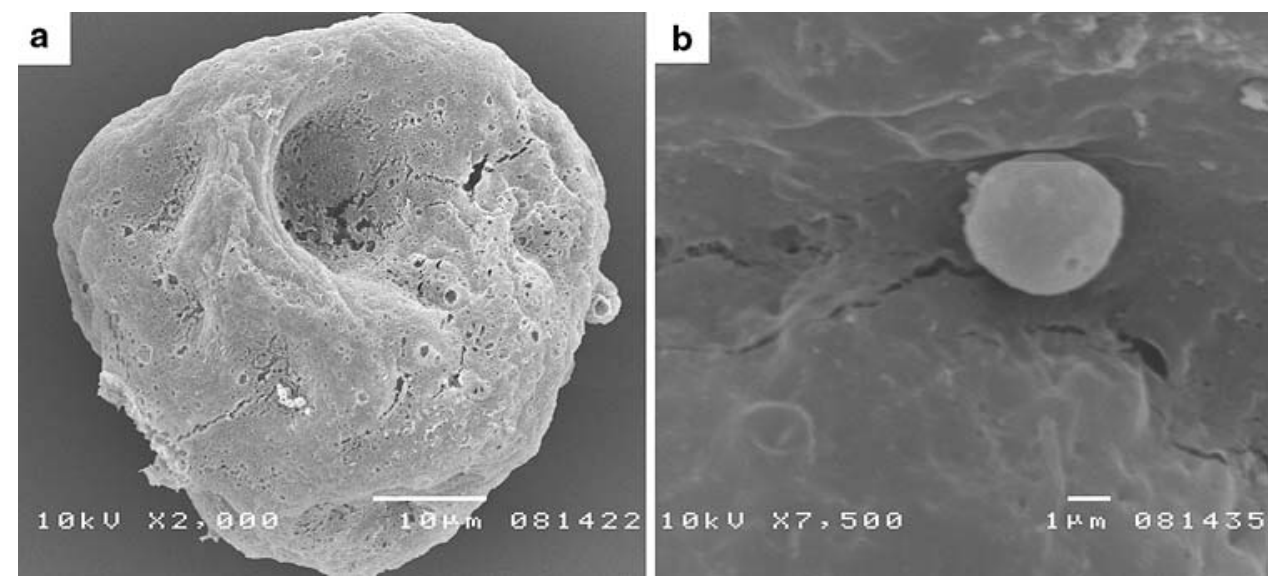

almost $5 \% / \mathrm{h}$ at its peak, against $0.6 \% / \mathrm{h}$ for the $20 \mu \mathrm{m}$ spheres over this time. This difference can be attributed to the difference in surface area per unit volume of the two particle sizes, allowing dexamethasone to diffuse faster from the smaller spheres due to the increased amount of polymer-water interfaces and decreased average path distance to solution. The possibility of some of the smaller spheres to start already undergoing degradation can not be excluded.

Additionally, by moving down with the size of the spheres, the proportion of released dexamethasone within the $550 \mathrm{~h}$ sampled was reduced from over $90-30 \%$.

In the papers by Lu et al. [9] and Sahoo et al. [12] it is reported that the surface of PLGA particles produced by the methods used in this paper are coated by a stable layer of PVA. Also, as the particle size decreases, the amount of PVA associated with the surface increases because of the increased surface area per unit volume allowing more PVA to collect onto the sphere surface during synthesis. Once in the release experiment this thickened layer of PVA (associated with smaller particles) may cause an exterior hydrogel to occur around the spheres, acting as a diffusion barrier for encapsulated drugs and limiting their total release. This could be minimised by further optimising the synthesis procedure. Figure $4 \mathrm{~b}$ shows a SEM image of the $1 \mu \mathrm{m}$ spheres after $550 \mathrm{~h}$ of degradation. It can be clearly seen that there are undegraded spheres still associated with PVA after this time. However, the amount of spheres still visible was significantly less than the initial quantity in the sample, suggesting that the $1 \mu \mathrm{m}$ spheres were entirely degraded when free in solution.

When comparing the two types of spheres in the view of a possible application for medical combination devices, the $20 \mu \mathrm{m}$ spheres may offer some advantages as they are less likely to be removed from the desired peri-implant area (for example in case they become detached from the device surface) or rapidly broken down by an immune response. The drug release profile makes them feasible for applications where a sustained release is needed over a longer period (in this case about 23 days). However, the drug loading efficiency is low.

The $1.0 \mu \mathrm{m}$ spheres show a much higher drug loading efficiency reducing the amount of polymer matrix needed to get the same amount of drug released. Being significantly smaller in size, this formulation can maintain more of the initial implant surface texture, and would have more surface area contact with the implant, reducing the probability of being removed physically from the implant surface. The system exhibited a burst release of the drug (about $20 \%$ in one day) and the total amount of drug released over the $550 \mathrm{~h}$ period represented only about $30 \%$ of the loaded dexamethasone. Therefore, further work has to be performed in order to increase the percentage of drug released and, when required, extend the duration of the release. Factors such as lactide:glycolide ratio $[3,6]$ and polymer molecular weight are affecting the rate of diffusion [7] and could be investigated in regard to prolonging drug release. In addition, investigation into the use of an alternative emulsifier should also be performed, as it is possible that the PVA used in this study could have contributed to the low total yield released from the $1.0 \mu \mathrm{m}$ spheres.

With regard to the effect of dexamethasone on osteoblast cells, previous work [11] has shown that a concentration of $10 \mu \mathrm{M}$ is suitable for differentiation over a period of 28 days. Both sizes of sphere easily produce a concentration of over $10 \mu \mathrm{M}$ within the primary layer of cells within the first hour after implantation. However, the larger, slower releasing spheres seem to be more suitable for inducing osteoblast differentiation, as it is important to maintain adequate concentrations to these cells over as much of the full period of 28 days as possible to induce a cellular response.

It has been shown [14] that a dose of $0.5 \mu \mathrm{g} / \mathrm{mm}^{2}$ is necessary for immunosuppression in the vicinity of bioimplants. With the spheres produced by these means such 
doses can be achieved. It would require a loading of about $0.05 \mathrm{mg} / \mathrm{mm}^{2}$ of the $20 \mu \mathrm{m}$ spheres, or only $0.005 \mathrm{mg} /$ $\mathrm{mm}^{2}$ of the $1 \mu \mathrm{m}$ spheres due to the increased drug loading efficiency of the smaller spheres. It is interesting to note that the fundamental immune response is primarily within the first few hours to days of implantation [1], and thus a high dose of dexamethasone would be advised through this period. This would be an excellent use of the $1 \mu \mathrm{m}$ spheres. Follow-up studies will be performed to investigate the drug release kinetics for such a system from a solid substrate.

\section{Conclusions}

Two different sizes (i.e. 20 and $1.0 \mu \mathrm{m}$ ) of PLGA spheres loaded with dexamethasone were produced by the oil-inwater emulsion/solvent evaporation method using two synthesis schemes. Their morphology, drug loading efficiency and drug release profile were investigated and compared. Dexamethasone release was monitored in phosphate buffer saline solution at $37^{\circ} \mathrm{C}$ for a period of $550 \mathrm{~h}$ using the HPLC analysis.

The results indicated formation of spheres with two different and reproducible size ranges, uniform shape and smooth outer surfaces. The different size of the produced spheres affected significantly the drug loading efficiency, the release profiles and the doze of the released drug. The $1.0 \mu \mathrm{m}$ formulation showed higher loading efficiency (11 vs. $1 \%$ ), a burst release of the drug in the first day and a maximum of about $30 \%$ release of the encapsulated dexamethasone until the end of the 23 days experiment. In comparison, the $20 \mu \mathrm{m}$ formulation exhibited an almost linear release profile with about $90 \%$ of the encapsulated drug being released by the end of the experiment. However, the drug loading efficiency was significantly lower. The main differences observed were explained taking into consideration the relatively higher amount of polymerwater interfaces generated during the synthesis of the $1.0 \mu \mathrm{m}$ spheres that enabled more dexamethasone to be encapsulated in the resultant spheres. Similarly, during the release phase, the larger surface area per unit volume favored faster water inclusion into the polymer and therefore easier drug diffusion and possible polymer scission.
Both formulations have attractive features for potential use in medical combination devices to control osteoblast differentiation and/or immune suppression in the periimplant area. In order to achieve both effects, a combined system of large and small spheres immobilized on the implant surface could be a solution.

Acknowledgements The support of Michel van der Brink from the Laboratory for Process Equipment (TU Delft) for the scanning electron microscopy investigations is greatly appreciated.

Open Access This article is distributed under the terms of the Creative Commons Attribution Noncommercial License which permits any noncommercial use, distribution, and reproduction in any medium, provided the original author(s) and source are credited.

\section{References}

1. J.M. Anderson, M.S. Shive, Adv. Drug Deliv. Rev. 28, 5 (1997). doi:10.1016/S0169-409X(97)00048-3

2. A.A. Ignatius, L.E. Claes, Biomaterials 17, 831 (1996). doi: 10.1016/0142-9612(96)81421-9

3. X.S. Wu, N. Wang, J. Biomater. Sci. Polym. Ed. 12, 21 (2001). doi: $10.1163 / 156856201744425$

4. T. Hickey, D. Kreutzer, D.J. Burgess, F. Moussy, J. Biomed. Mater. Res. 61, 180 (2002). doi:10.1002/jbm.10016

5. U. Bilati, E. Allemann, E. Doelker, Eur. J. Pharm. Biopharm. 59, 375 (2005). doi:10.1016/j.ejpb.2004.10.006

6. G.B. Wei, G.J. Pettway, L.K. McCauley, P.X. Ma, Biomaterials 25, 345 (2004). doi:10.1016/S0142-9612(03)00528-3

7. S. Jaraswekin, S. Prakongpan, R. Bodmeier, J. Microencapsul. 24, 117 (2007). doi:10.1080/02652040701233655

8. T. Hickey, D. Kreutzer, D.J. Burgess, F. Moussy, Biomaterials 23, 1649 (2002). doi:10.1016/S0142-9612(01)00291-5

9. J. Liu, S.M. Zhang, P.P. Chen, L. Cheng, W. Zhou, W.X. Tang, Z.W. Chen, C.M. Ke, J. Mater. Sci. Mater. Med. 18, 2205 (2007). doi:10.1007/s10856-007-3010-0

10. D.H.R. Kempen, L. Lu, T.E. Hefferan, L.B. Creemers, A. Maran, K.L. Classic, W.J.A. Dhert, M.J. Yaszemski, Biomaterials 29, 3245 (2008). doi:10.1016/j.biomaterials.2008.04.031

11. M. Jager, J. Fischer, W. Dohrn, X.N. Li, D.C. Ayers, A. Czibere, W.C. Prall, S. Lensing-Hohn, R. Krauspe, J. Orthop. Res. 26, 1440 (2008). doi:10.1002/jor.20565

12. S.K. Sahoo, J. Panyam, S. Prabha, V. Labhasetwar, J. Control. Release 82, 105 (2002). doi:10.1016/S0168-3659(02)00127-X

13. C. Gomez-Graete, N. Tsapis, M. Besnard, A. Bochot, E. Fattal, Int. J. Pharm. 331, 153 (2007). doi:10.1016/j.ijpharm.2006.11.028

14. B.S.F. Santiago Jimenez-Valero, Catheter. Cardiovasc. Interv. 70, 492 (2007). doi:10.1002/ccd.21131 